\title{
Revista Brasileira de Enfermagem REBEn Elaboração e validação do instrumento de entrevista de enfermagem
}

\author{
Elaboration and validation of the instrument nursing interview \\ Elaboración y validación del instrumento de la entrevista de enfermería
}

\section{Patrícia Madalena Vieira Hermida}

Mestre em Enfermagem pela Universidade Estadual de Campinas. Especialista em Formação Profissional na Área da Saúde - Enfermagem, Enfermeira do Programa de Saúde da Familia. patymadale@yahoo.com.br

Izilda Esmênia Muglia Araújo

Enfermeira, Doutora e Professora Assistente do Departamento de Enfermagem da FCM - UNICAMP; Orientadora deste estudo.

Parte da Dissertação de Mestrado apresentada à UNICAMP, 2005..
Submissão: 15/04/2005

Aprovação: 05/05/2006

\section{RESUMO}

Esta pesquisa teve como objetivos elaborar e validar o instrumento Entrevista de Enfermagem para a coleta de dados na assistência de enfermagem, guiado pelo referencial de Orem. Para validar o conteúdo do instrumento e de seu respectivo guia de apoio à decisão, estes foram encaminhados para apreciação de juízes, cuja concordância entre eles, analisada pelo Teste Qui-Quadrado de Cochran, apontou diferença estatisticamente significativa quanto à objetividade do instrumento, porém, o número de respostas positivas foi maior, indicando que o instrumento apresenta os quesitos pertinentes. A análise descritiva da concordância quanto ao guia de apoio à decisão, mostrou que apenas a objetividade obteve $50 \%$ de respostas negativas, sugerindo alterações. Concluindo, o instrumento apresenta validade permitindo coletar dados de forma sistemática e voltados ao autocuidado.

Descritores: Coleta de dados; Estudos de validação; Enfermagem.

\section{ABSTRACT}

The purpose of this research was to elaborate and validate the instrument Nursing Interview to collect data related to nursing care based on the Orem theory. The contents of this instrument and the supporting guidelines were referred to referees who analyzed the concordance among them by applying Cochran's Chi -Square test, which revealed a statistically significant difference regarding objectivity but the great number of positive answers indicated that the instrument also dealt with pertinent aspects. The descriptive analysis of concordance related to the supporting guidelines demonstrated that only objectivity obtained $50 \%$ of negative responses, suggesting that changes were required. Finally, we may conclude that this instrument collects data related to self- care in a systematic manner.

Descriptors: Data collection; Validation studies; Nursing.

\section{RESUMEN}

Esta pesquisa fue ejecutada con el objetivo de elaborar y validar el instrumento Entrevista de Enfermería para la colecta de datos en la asistencia de enfermería, guiado por los postulados de Orem. Para validar el contenido del instrumento y de su respectiva guía de apoyo para la decisión, estos fueron encaminados para la apreciación de jueces, cuyas concordancias analizadas por el Test de Cochran, determinaron diferencias significativas estadísticamente en lo que se refiere a la objetividad del instrumento, pero, el número de respuestas positivas fue mayor, indicando que el instrumento representó los requisitos pertinentes. El análisis descriptivo de concordancia referente a la guía de apoyo a la decisión, mostró solamente una objetividad de $50 \%$ de respuestas negativas, indicando la necesidad de alteraciones. En conclusión, el instrumento presenta validez, permitiendo colectar datos de forma sistemática y orientados al autocuidado.

Descriptores: Recolección de datos, Estudios de validación; Enfermería.

Hermida PMV, Araújo IEM. Elaboração e validação do instrumento de entrevista de enfermagem. Rev Bras Enferm 2006 maio-jun; 59(3): 314-20.

\section{INTRODUÇÃO}

O desenvolvimento do conhecimento de enfermagem baseado em teorias próprias, deu origem ao Processo de Enfermagem $(\mathrm{PE})$ que recebe várias definições de acordo com muitos autores e teóricas de enfermagem. Em cada teoria, ele segue um modelo específico e é fundamentado de acordo com os conceitos, pressupostos e proposições próprios dessas teorias. Pode ser entendido como a aplicação prática de uma teoria de enfermagem no cotidiano da assistência de enfermagem.

Para Orem (1) "o processo de enfermagem é um termo usado pelas enfermeiras ao referirem-se às operações profissionais-tecnológicas da prática da enfermagem e ao planejamento e operações 
avaliatórias associadas". É uma ação regular e contínua ou uma sucessão de ações que ocorrem de maneira definida.

A escolha do modelo teórico e a adaptação da teoria à realidade que se pretende aplicar, são apontados por alguns autores ${ }^{(2,3)}$. Um deles escreve que, "O modelo teórico escolhido deve atender ao perfil epidemiológico da clientela, direcionando a assistência de enfermagem para 0 atendimento ambulatorial, de emergência ou de uma unidade de internação"(3).

O PE vem sendo estudado por muitos autores e possui aplicações tanto no âmbito nacional como no exterior. No Brasil, Wanda de Aguiar Horta baseada na Teoria das Necessidades Humanas Básicas, foi a precursora do PE nos anos $70^{(4)}$.

O PE é composto por fases que diferem entre si de acordo com cada modelo teórico e diversos autores. A fase da coleta de dados é apontada como a base fundamental para o desenvolvimento da SAE e todo o planejamento da assistência depende da objetividade, fidedignidade e abrangência com que os dados iniciais são coletados ${ }^{(5)}$. Essa fase tem recebido diversas denominações: levantamento de dados, avaliação, histórico de enfermagem e anamnese de enfermagem, esta última se refere à primeira terminologia atribuída a essa fase, no Brasil(4,6,7).

Nesse momento do texto, convém destacar e discutir como o termo Histórico de Enfermagem vem sendo utilizado. Para alguns autores, Histórico de Enfermagem e Exame Físico são etapas distintas do PE. A exemplo, o próprio Conselho Federal de Enfermagem os considera dessa forma, segundo a Resolução 272/2002 ${ }^{(8)}$. Segundo outros autores, o Exame Físico juntamente com a Entrevista de Enfermagem, constituem o Histórico de Enfermagem ${ }^{(3,4,9,10)}$. Essas aplicações distintas do termo Histórico de Enfermagem apontam a necessidade de se refletir a definição e delimitação dessa terminologia, para que a comunicação entre os profissionais de enfermagem seja uniforme, coerente e compreensível.

No PE a fase de coleta de dados é definida por alguns autores ${ }^{(4,6,11)}$. A análise dos conceitos de vários autores acerca da terminologia "Histórico de Enfermagem", apontou que há uma predominância em defini-lo como a primeira fase de um processo, no qual coleta-se dados do paciente, e estes, após analisados, permitem identificar problemas e determinar o diagnóstico de enfermagem, fornecendo, conseqüentemente, subsídios para elaborar o plano de cuidados $^{(7)}$.

Alguns autores apresentam quatro tipos de dados coletados nessa primeira fase do PE: dados subjetivos, objetivos, históricos e atuais. Estes podem ser obtidos, utilizando-se: a entrevista, a observação, o exame físico, os resultados de provas diagnósticas, a revisão de do prontuário e a colaboração de outros profissionais(5).

\section{REFERENCIAL TEÓRICO}

Para consolidar a proposta deste estudo, primeiramente, definiu-se o referencial teórico que o sustentaria, optando-se pela teoria de Dorothea E. Orem.

\subsection{A Teoria}

A teoria geral de Orem, denominada Teoria do Déficit de Autocuidado de Enfermagem, é constituída por três teorias interrelacionadas (construtores teóricos): teoria do autocuidado; do déficit do autocuidado e, dos sistemas de enfermagem ${ }^{(1)}$.

\subsection{Componentes Funcionais}

Dentre os componentes funcionais da teoria, a metodologia será destacada, uma vez que, neste estudo, o instrumento Entrevista de Enfermagem elaborado e testado quanto à validade, poderá ser utilizado como uma estratégia metodológica para a assistência de enfermagem.

\subsubsection{Metodologia}

O PE pode ser entendido como a metodologia ou o modelo pelo qual a teoria deve ser aplicada pela enfermagem. No modelo de Orem o PE segue três passos:

$1^{0}$ - Diagnóstico de enfermagem e prescrição - determina as necessidades ou não dos cuidados de enfermagem. 0 enfermeiro realiza a coleta de dados, reunindo esses dados conforme os requisitos de autocuidado (universais, desenvolvimentais, desvios de saúde). Também se analisa e inter-preta dados, fazendo julgamentos;

$2^{\circ}$ - Esboço de um sistema de enfermagem e de um plano para o fornecimento de atendimento - é a fase do planejamento dos sistemas de enfermagem e do planejamento da execução das ações de enfermagem, de acordo com a demanda de autocuidado do indivíduo identificada na etapa do diagnóstico;

$3^{\circ}$ - Produção e controle dos sistemas de enfermagem (planejamento e controle) - a enfermagem realiza os cuidados ao indivíduo ou o auxilia; inclui a avaliação dos cuidados prestados e a evolução do paciente perante esses cuidados.

Para melhor compreensão do modelo do PE proposto por Orem, no Quadro 1 estão representadas suas fases, comparando-as com as de outros autores ${ }^{(12)}$.

Quanto ao referencial teórico adotado, convém ressaltar que:

- uma justificativa relevante para este estudo, está no fato de não ter sido encontrada, após vasto levantamento bibliográfico no sistema Bireme, nenhuma pesquisa direcionada para os pacientes neurocirúrgicos utilizando Orem;

- neste estudo os requisitos de autocuidado, um dos conceitos do referencial de Orem, estão presentes no instrumento Entrevista de Enfermagem, orientando os questionamentos e observações dos enfermeiros;

- optou-se por desenvolver a coleta de dados por meio do instrumento Entrevista de Enfermagem, compreendendo-se que a coleta de dados faz parte do primeiro passo da fase intelectual do PE de Orem.

\section{OBJETIVOS}

Elaborar o instrumento Entrevista de Enfermagem como uma das etapas da SAE, respaldando-se no referencial teórico de Dorothea Orem e, avaliar a validade de conteúdo deste instrumento.

\section{SUJEITOS E MÉTODOS}

\subsection{Delineamento do estudo}

O presente estudo é do tipo pesquisa de desenvolvimento, estratégia

\begin{tabular}{|c|c|c|}
\hline Fases do PE & Orem & Outros autores \\
\hline Fase & $\begin{array}{l}1^{\text {a }} \text { passo - Coleta de dados; determinar porque um paciente precisa do cuidado de enfermagem; } \\
\text { considerar a história e estilo de vida do paciente. }\end{array}$ & $\begin{array}{c}1^{\mathrm{a}} \text { - Avaliação } \\
2^{\mathrm{a}} \text { - Diagnóstico }\end{array}$ \\
\hline Intelectual & $\begin{array}{l}2^{\mathrm{a}} \text { passo - projetar um sistema de enfermagem para o paciente; planejar para a independência do } \\
\text { cuidado de enfermagem. }\end{array}$ & $3^{\mathrm{a}}$ - Planejamento \\
\hline Fase Prática & $\begin{array}{l}3^{\text {a }} \text { passo - Iniciar, conduzir e controlar as ações necessárias para o cuidado de enfermagem. } \\
\text { Controlar a independência do cuidado de enfermagem. }\end{array}$ & $\begin{array}{l}4^{\mathrm{a}} \text { - Implementação } \\
5^{\mathrm{a}} \text { - Avaliação }\end{array}$ \\
\hline
\end{tabular}

Quadro 1. Modelo do processo de enfermagem de Orem e de outros autores. 
de pesquisa que, utilizando sistematicamente os conhecimentos existentes, objetiva elaborar uma nova intervenção, ou melhorar consideravelmente uma intervenção existente ou, ainda, elaborar ou melhorar um instrumento, um dispositivo ou um método de medição(13)a . Este estudo foi dividido em duas etapas:

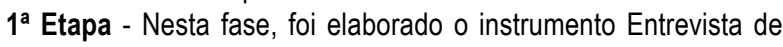
Enfermagem, com o objetivo de coletar os dados de pacientes internados.

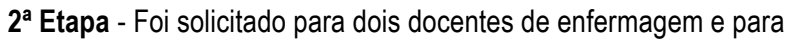
dois enfermeiros com experiência na área, para realizarem a validação do conteúdo.

\subsection{Procedimento}

Procurou-se, inicialmente, descrever os aspectos relacionados à elaboração do instrumento e, em seguida, os procedimentos metodológicos da sua validação.

\subsubsection{Instrumento}

\section{a) Elaboração do instrumento}

O instrumento foi elaborado com base na Teoria de Orem, em outros instrumentos e publicações sobre o tema e, na experiência assistencial das pesquisadoras. Para orientar o preenchimento do mesmo elaborouse o Guia de Apoio à Decisão.

Essa etapa de elaboração do instrumento exigiu, primeiramente, um levantamento bibliográfico para identificar trabalhos que utilizassem 0 referencial teórico de Orem e/ou instrumentos de coleta de dados, especialmente aqueles baseados nesse referencial. Para tanto, foram acessadas as bases de dados do LILACS, MEDLINE e BDENF da Bireme, utilizando os descritores: sistematização, assistência e enfermagem. A leitura dos resumos dos estudos apontados nesse levantamento possibilitou selecionar principalmente os que se referiam ao uso de instrumentos de coleta de dados e/ou à aplicação do referencial de Orem.

A partir daí, as pesquisadoras reuniram alguns instrumentos encontrados na literatura e estabeleceram as principais características do instrumento que seria elaborado para este estudo. Nesse momento, definiu-se que o foco da coleta de dados seria identificar os déficits de autocuidado, a partir do levantamento dos requisitos de autocuidado. Uma vez isto definido, foram selecionados e escritos tais requisitos no instrumento Entrevista de Enfermagem.

Baseado em Orem, o instrumento possui quatro categorias, na sequência: Fatores Pessoais e Condicionantes Básicos; Requisitos de Autocuidado nos Desvios de Saúde; Requisitos de Autocuidado Desenvolvimentais e Requisitos de Autocuidado Universais.

Os Fatores Pessoais e Condicionantes Básicos compõem-se de: nome do paciente, número de registro no hospital, procedência, idade, estado civil, filhos, sexo, naturalidade, data de nascimento, escolaridade, profissão, ocupação atual, etnia, unidade de internação procedente, diagnóstico médico atual, quarto, leito, data, hora e médico responsável. Estes dados permitem caracterizar a população estudada e realizar trabalhos futuros.

A categoria Requisitos de Autocuidado nos Desvios de Saúde é constituída pelos itens: queixa principal; início das queixas; evolução do problema; manifestações associadas; o que fez ao perceber o problema; exames realizados; tratamentos convencionais já realizados; terapêutica complementar usada. Todas essas informações devem ser descritas por extenso, pois tratam-se de itens abertos, importantes na compreensão do problema atual de saúde do paciente.

Nos Requisitos de Autocuidado Desenvolvimentais são apresentados

a O.C.D.E. La mesure des activités scientifiques et techniques, Manual de Frascati, Organisation de coopération et de développement économiques, Paris, 1980. os itens antecedentes pessoais e antecedentes familiares. Dentro do primeiro item incluem-se os subitens: imunizações; doenças; outras doenças ou informações; cirurgias realizadas; hospitalizações; medicamentos já utilizados. Quanto aos antecedentes familiares, o instrumento questiona os subitens: Causa Mortis de pais/irmãos, ocorrência de doenças na família e, outras doenças ou informações. Os subitens desta categoria permitem respostas abertas e/ou fechadas.

A categoria Requisitos de Autocuidado Universais permite identificar as características fisiopatológicas do paciente e as necessidades de autocuidado relacionadas. Nesta categoria estão os itens: padrões de sono e repouso; exercícios físicos; hábitos; padrões nutricionais/hidratação; atividade diária; participação ou dependência familiar; relacionamento familiar; espiritualidade; segurança emocional; higiene pessoal e, informações adicionais (espaço destinado à informações julgadas necessárias e que não podem ser registradas em nenhuma outra parte do instrumento). Esses itens admitem tanto respostas abertas como fechadas.

\section{b) Avaliação da validade do instrumento}

A validade é um dos critérios capaz de avaliar a qualidade de um instrumento ${ }^{(13,14)}$. Ela pode ser definida como a capacidade de um instrumento medir com precisão o que se propõe a medir, ou seja, o fenômeno estudado(13-15).

Existem três tipos principais de validade: validade de conteúdo, de construto e relacionada a um critério. A validade de conteúdo refere-se à análise minuciosa do conteúdo do instrumento, com objetivo de verificar se os itens propostos constituem-se numa amostra represen-tativa do assunto que se deseja medir. Nesse tipo de validação, os instrumentos são submetidos à apreciação de peritos no assunto, os quais podem sugerir a retirada, acréscimo ou modificação dos itens ${ }^{(13-15)}$.

Para proceder à validade de conteúdo neste estudo, optou-se em submeter o instrumento e respectivo Guia de Apoio à Decisão à apreciação de quatro juízes com experiência na área, sendo dois docentes de enfermagem de duas universidades públicas, uma do Estado de São Paulo e outra de Santa Catarina, e dois enfermeiros assistenciais, um atuando em hospital público e o outro em hospital privado, ambos do Estado de São Paulo. Os juízes foram selecionados pelas competências:

- docente com titulação de doutor, experiência em assistência de enfermagem ao paciente neurocirúrgico e dissertação de mestrado em sistematização da assistência de enfermagem em terapia intensiva.

- docente com titulação de doutor, domínio acerca da organização e de metodologias assistenciais.

-enfermeiro e mestrando em enfermagem, com experiência em assistência de enfermagem ao paciente neurocirúrgico;

- enfermeiro e mestre em enfermagem, com dissertação baseada em Orem.

Para que o instrumento Entrevista de Enfermagem e seu respectivo guia pudessem ser apreciados pelos juízes, foram elaborados dois instrumentos de avaliação, constituídos pelos itens do instrumento e pelos critérios de avaliação: organização, objetividade, clareza, facilidade de leitura e compreensão do conteúdo. Cada item foi avaliado com relação a estes cinco critérios, para os quais os juízes deveriam responder sim ou não. Vale esclarecer que, como não foi especificado para os juízes a definição dos termos utilizados como critérios de avaliação, cada juíz adotou seus próprios conceitos acerca dos mesmos para avaliar cada item.

No final destes instrumentos de avaliação dos juízes existiam espaços em aberto para que fossem registrados: itens necessários, porém ausentes no instrumento; itens desnecessários no instrumento e, comentários e/ou sugestões.

As avaliações dos juízes retornaram às pesquisadoras após dois 
meses, com a análise dos itens do instrumento, sugestões de alterações, de acréscimos e também de referências bibliográficas.

\subsection{Aspectos éticos da pesquisa}

O projeto foi aprovado pelo Comitê de Ética em Pesquisa da Faculdade de Ciências Médicas - FCM, da UNICAMP, sob parecer nº 290/2003.

\subsection{Tratamento e análise estatística dos dados}

Os dados obtidos foram tabulados eletronicamente com o auxílio do programa Microsoft Excel - XP e analisados quantitativamente sob orientação do Serviço de Estatística da Comissão de Pesquisa da FCM - Unicamp.

Para analisar a concordância das respostas dos juízes, quando da validação de conteúdo do instrumento Entrevista de Enfermagem, para todos os itens do instrumento foi utilizado o Teste Qui-Quadrado de Cochran para respostas dicotômicas. O nivel de significância considerado foi de $5 \%(p<0,05)$. Para a análise de concordância das respostas dos juízes para o Guia de Apoio à Decisão, realizou-se a análise da freqüência das respostas (sim ou não) bem como a análise descritiva das sugestões de alterações dos juízes.

Para incorporação das sugestões dos juízes no instrumento e respectivo guia, foi considerada a porcentagem obtida em cada item. Com isso, o item que obteve pontuação total menor que $75 \%$ para qualquer um dos critérios avaliados, foi alterado ou excluído do instrumento. Entretanto, mesmo quando o item recebeu pontuação total acima de $75 \%$, mas algum dos juízes deferiu pontuação menor que $75 \%$ foi considerada a justificativa/sugestão do juiz para alteração.

As respostas dos juízes receberão análise descritiva expressa por tabelas e figuras. Na avaliação do instrumento Entrevista de Enfermagem, os itens analisados serão representados por letras que corresponderão a: Identificação (A); Histórico da Patologia atual (B); Histórico da Saúde (C); Padrões de Sono e Repouso (D); Exercícios Físicos (E); Vícios (F); Padrões Nutricionais/Hidratação (G); Estilo de Vida (H); Relacionamento Familiar (I); Espiritualidade (J); Ansiedade (K); Depressão (L); Comportamento Emocional (M) e, Higiene Pessoal (N).

$\mathrm{Na}$ avaliação do Guia de Apoio à Decisão do instrumento, realizada pelos juizes, as respostas estarão representadas por meio de figuras, nas quais os números 1, 2, 3, 4 e 5 corresponderão aos critérios organização, objetividade, clareza, facilidade de leitura e compreensão de conteúdo, respectivamente.

\section{RESULTADOS}

\subsection{Instrumento Entrevista de Enfermagem}

Na Tabela 1 encontram-se as respostas da avaliação do instrumento Entrevista de Enfermagem quanto à organização, não havendo discordância entre os juízes ( $p=0,087$ ), uma vez que foram observadas um total de $83,9 \%$ de respostas positivas. Entretanto, o resultado de $64,3 \%$ de respostas positivas apontado pelo juiz $n^{0} 1$, sugeriu a necessidade de rever alguns itens do instrumento. As modificações realizadas no instrumento para melhor adequá-lo ao aspecto da organização estão relacionadas à mudança na seqüência de apresentação dos subitens, acréscimo de subitens, troca de terminologias e a numeração de todos os itens e subitens.

A Tabela 2 apresenta os resultados da avaliação da objetividade do instrumento, mostrando que houve discordância significativa entre as respostas $(p<0,001)$, ocorrida especialmente pelo número de respostas negativas do juiz $n^{0} 1$, embora o total de respostas positiva tenha sido $76,9 \%$, valor este que obedece ao critério de validação deste instrumento. Tal discordância sugeriu a necessidade de rever alguns itens apontados pelos juízes $n^{0} 1$ e $n^{0} 2$.

Para melhor visualizar os itens que influenciaram tal discordância, 0 Gráfico 1 representa a freqüência das respostas dos juízes para os quatorze itens avaliados, os quais se encontram identificados pelas letras de $\mathrm{A}$ a $\mathrm{N}$. Observa-se que os itens com maior número de respostas negativas foram: Estilo de Vida, representado pela letra $\mathrm{H}$; Ansiedade, (K) e, Depressão, (L), todos com 50\%.

Considerando-se que o maior número de respostas negativas foram atribuídas aos itens Estilo de Vida, Ansiedade e Depressão, conforme 0 Gráfico 1, as modificações mais relevantes sugeridas pelos juízes e acatadas pelas pesquisadoras para melhorar a objetividade do instrumento, foram a exclusão destes itens, com a reelaboração e a substituição do item Estilo de Vida pelos itens Atividade Diária e Participação ou Dependência Familiar e, a Ansiedade e Depressão substituídos por um novo item denominado Segurança Emocional. A definição e delimitação deste, permitiu inclusive a exclusão do item Comportamento Emocional. As demais modificações foram menos significativas.

$\mathrm{Na}$ Tabela 3 as respostas mostram que houve concordância entre os juízes quanto à clareza dos itens $(p=0,095)$ com um total de $75 \%$ de respostas positivas, porém houve necessidade de rever itens, indicados principalmente pelo juiz $n^{0} 1$.

A maioria das modificações realizadas para melhorar a clareza do instrumento está relacionada a: descrição por extenso de palavras abreviadas e de siglas técnicas, exclusão de palavras desnecessárias, troca de algumas terminologias e reescrita de alguns subitens para evitar dúbio sentido. Além disso, foram excluídos os itens Ansiedade e Depressão, como já mencionado.

A Tabela 4 mostra que houve concordância entre as respostas dos juízes em relação à facilidade de leitura dos itens $(p=0,223)$ e um total de respostas afirmativas de $91,1 \%$, mesmo assim, os itens que receberam respostas negativas foram revistos e as alterações sugeridas pelos juizes e acatadas pelas pesquisadoras são referentes a: especificação de

Tabela 1. Distribuição do total de respostas dos juízes na avaliação do instrumento Entrevista de Enfermagem, quanto à sua organização. Campinas, 2004.

\begin{tabular}{|c|c|c|c|c|c|c|c|c|c|c|}
\hline \multirow{2}{*}{$\mathrm{N}^{0}$ Respostas } & \multicolumn{2}{|c|}{ Juiz $n^{0} 1$} & \multicolumn{2}{|c|}{ Juiz $n^{0} 2$} & \multicolumn{2}{|c|}{ Juiz $n^{0} 3$} & \multicolumn{2}{|c|}{ Juiz $n^{0} 4$} & \multicolumn{2}{|c|}{ Total } \\
\hline & $n$ & $\%$ & $\mathrm{n}$ & $\%$ & $\mathrm{n}$ & $\%$ & $\mathrm{~N}$ & $\%$ & $\mathrm{~N}$ & $\%$ \\
\hline Sim & 9 & 64,3 & 11 & 78,6 & 13 & 92,9 & 14 & 100 & 47 & 83,9 \\
\hline Não & 5 & 35,7 & 3 & 21,4 & 1 & 7,1 & - & - & 9 & 16,1 \\
\hline
\end{tabular}

$p=0,087$

Tabela 2. Distribuição do total de respostas dos juízes, quanto à objetividade, na avaliação do instrumento Entrevista de Enfermagem. Campinas, 2004.

\begin{tabular}{|c|c|c|c|c|c|c|c|c|c|c|}
\hline \multirow{2}{*}{$N^{0}$ Respostas } & \multicolumn{2}{|c|}{ Juiz $n^{0} 1$} & \multicolumn{2}{|c|}{ Juiz $n^{0} 2$} & \multicolumn{2}{|c|}{ Juiz $n^{0} 3$} & \multicolumn{2}{|c|}{ Juiz $n^{0} 4$} & \multicolumn{2}{|c|}{ Total } \\
\hline & $n$ & $\%$ & $\mathrm{n}$ & $\%$ & $\mathrm{n}$ & $\%$ & $\mathbf{N}$ & $\%$ & $\mathrm{~N}$ & $\%$ \\
\hline Sim & 4 & 28,6 & 11 & 78,6 & 14 & 100,0 & 14 & 100,0 & 43 & 76,9 \\
\hline Não & 10 & 71,4 & 3 & 21,4 & - & - & - & - & 13 & $23,1^{*}$ \\
\hline Total & 14 & 100,0 & 14 & 100,0 & 14 & 100,0 & 14 & 100,0 & 56 & 100,0 \\
\hline
\end{tabular}




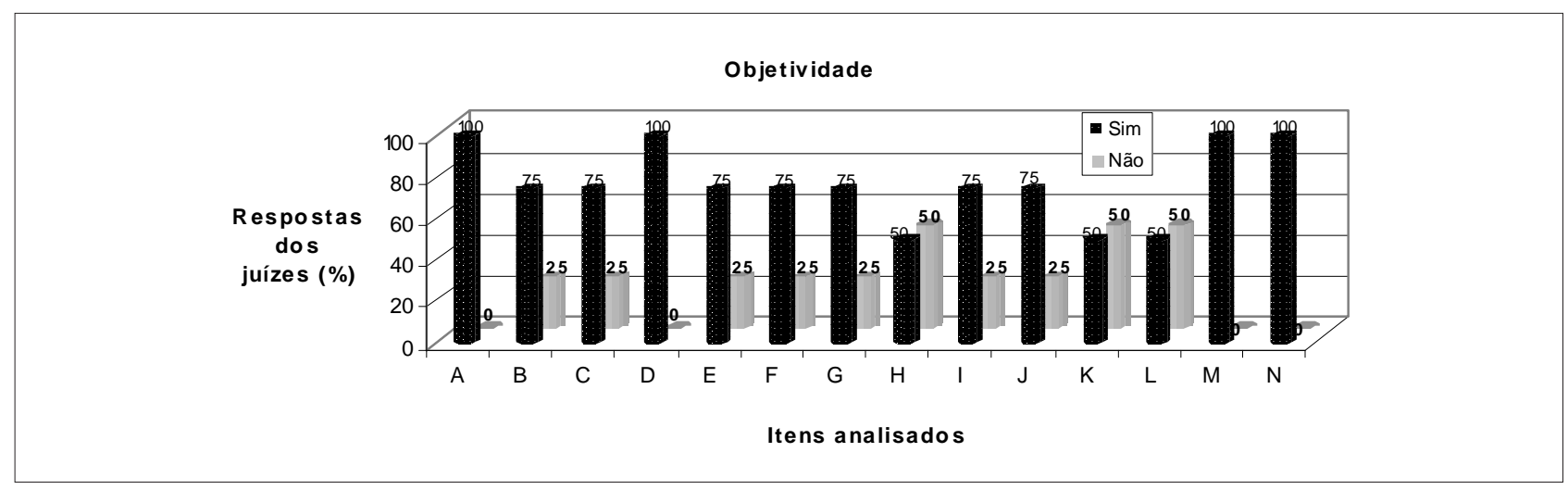

Gráfico 1 - Distribuição da freqüência relativa das respostas dos juízes em relação à objetividade dos itens da Entrevista de Enfermagem. Campinas, 2004.

Tabela 3. Distribuição do total de respostas dos juízes na avaliação do instrumento Entrevista de Enfermagem, quanto à sua clareza. Campinas, 2004.

\begin{tabular}{|c|c|c|c|c|c|c|c|c|c|c|}
\hline \multirow{2}{*}{$N^{0}$ Respostas } & \multicolumn{2}{|c|}{ Juiz $\mathrm{n}^{0} 1$} & \multicolumn{2}{|c|}{ Juiz $n^{0} 2$} & \multicolumn{2}{|c|}{ Juiz $n^{0} 3$} & \multicolumn{2}{|c|}{ Juiz $n^{0} 4$} & \multicolumn{2}{|c|}{ Total } \\
\hline & $\mathrm{n}$ & $\%$ & $\mathrm{n}$ & $\%$ & $\mathrm{n}$ & $\%$ & $\mathrm{~N}$ & $\%$ & $\mathrm{~N}$ & $\%$ \\
\hline Sim & 7 & 50 & 11 & 78,6 & 12 & 85,7 & 12 & 85,7 & 42 & 75 \\
\hline Não & 7 & 50 & 3 & 21,4 & 2 & 14,3 & 2 & 14,3 & 14 & 25 \\
\hline Total & 14 & 100 & 14 & 100 & 14 & 100 & 14 & 100 & 56 & 100 \\
\hline
\end{tabular}

$p=0,095$

Tabela 4. Distribuição do total de respostas dos juízes quanto à facilidade de leitura na avaliação do instrumento Entrevista de Enfermagem. Campinas, 2004.

\begin{tabular}{|c|c|c|c|c|c|c|c|c|c|c|}
\hline \multirow{2}{*}{$\mathrm{N}^{0}$ Respostas } & \multicolumn{2}{|c|}{ Juiz $n^{0} 1$} & \multicolumn{2}{|c|}{ Juiz $n^{0} 2$} & \multicolumn{2}{|c|}{ Juiz $n^{0} 3$} & \multicolumn{2}{|c|}{ Juiz $n^{0} 4$} & \multicolumn{2}{|c|}{ Total } \\
\hline & $\mathrm{n}$ & $\%$ & $n$ & $\%$ & $\mathrm{n}$ & $\%$ & $\mathrm{~N}$ & $\%$ & $\mathrm{~N}$ & $\%$ \\
\hline Sim & 14 & 100 & 11 & 78,6 & 13 & 92,9 & 13 & 92,9 & 51 & 91,1 \\
\hline Não & - & - & 3 & 21,4 & 1 & 7,1 & 1 & 7,1 & 5 & 8,9 \\
\hline Total & 14 & 100 & 14 & 100 & 14 & 100 & 14 & 100 & 56 & 100 \\
\hline
\end{tabular}

$p=0,223$

Tabela 5. Distribuição do total de respostas dos juízes quanto à compreensão do conteúdo na avaliação da Entrevista de Enfermagem. Campinas, 2004.

\begin{tabular}{|c|c|c|c|c|c|c|c|c|c|c|}
\hline \multirow{2}{*}{$N^{0}$ Respostas } & \multicolumn{2}{|c|}{ Juiz $n^{0} 1$} & \multicolumn{2}{|c|}{ Juiz $n^{0} 2$} & \multicolumn{2}{|c|}{ Juiz $n^{0} 3$} & \multicolumn{2}{|c|}{ Juiz $n^{0} 4$} & \multicolumn{2}{|c|}{ Total } \\
\hline & $\mathrm{n}$ & $\%$ & $\mathrm{n}$ & $\%$ & $\mathrm{n}$ & $\%$ & $\mathrm{~N}$ & $\%$ & $\mathrm{~N}$ & $\%$ \\
\hline Sim & 08 & 57,1 & 11 & 78,6 & 13 & 92,9 & 12 & 85,7 & 44 & 78,6 \\
\hline Não & 06 & 42,9 & 3 & 21,4 & 1 & 7,1 & 2 & 14,3 & 12 & 21,4 \\
\hline Total & 14 & 100,0 & 14 & 100,0 & 14 & 100,0 & 14 & 100,0 & 56 & 100,0 \\
\hline
\end{tabular}

$p=0,112$

abreviaturas no guia, substituição de siglas pelo seu significado escrito por extenso e a exclusão dos itens Ansiedade e Depressão.

A Tabela 5 demonstra a concordância entre as respostas dos juízes quanto à compreensão do conteúdo dos itens do instrumento $(p=0,112)$, porém, a avaliação do juiz $n^{0} 1$ representada por $42,9 \%$ de respostas negativas, sugeriu a necessidade de rever alguns itens, mesmo tendo na totalidade $78,6 \%$ de respostas positivas.

As modificações realizadas para melhorar a compreensão do conteúdo tratam-se de alterações descritas anteriormente, sendo a mais significativa a exclusão dos itens Ansiedade e Depressão, substituindo-os pelo item Segurança Emocional com definição própria bem caracterizada no Guia de Apoio à Decisão.

Do total de avaliações do instrumento Entrevista de Enfermagem, realizada pelos quatro juízes, pode ser observado que o maior número de respostas negativas foram apresentadas pelo juiz $n^{0} 1 \mathrm{e}$, atendendo o critério adotado foi considerada a justificativa/sugestão do juiz para alteração dos itens.

\subsection{Guia de Apoio à Decisão}

O Gráfico 2 apresenta a freqüência das respostas dos juízes quanto à organização, objetividade, clareza, facilidade de leitura e compreensão do conteúdo, representados respectivamente pelos números 1, 2, 3, 4 e 5, observados na avaliação do Guia de Apoio à Decisão da Entrevista de Enfermagem. A objetividade foi o único item que obteve $50 \%$ de respostas negativas, enquanto os demais receberam $75 \%$ de respostas afirmativas.

As sugestões dos juízes foram analisadas e o guia foi submetido à alterações, como: síntese; conceituação/definição de termos, como atividade diária, segurança emocional, medo, ansiedade e desesperança; descrição por extenso dos níveis de escolaridade e descrição dos sintomas de medo, ansiedade e desesperança.

\section{DISCUSSÃO}

O instrumento Entrevista de Enfermagem teve uma avaliação positiva em relação à organização, objetividade, clareza, facilidade de leitura e compreensão do conteúdo, respectivamente, representada por $83,9 \%$, $76,9 \%, 75 \%, 91,1 \%$ e $78,6 \%$, sugerindo que é organizado, objetivo, claro, fácil de ler e ser compreendido. As sugestões dos juízes foram incorporadas para o aperfeiçoamento do instrumento. 


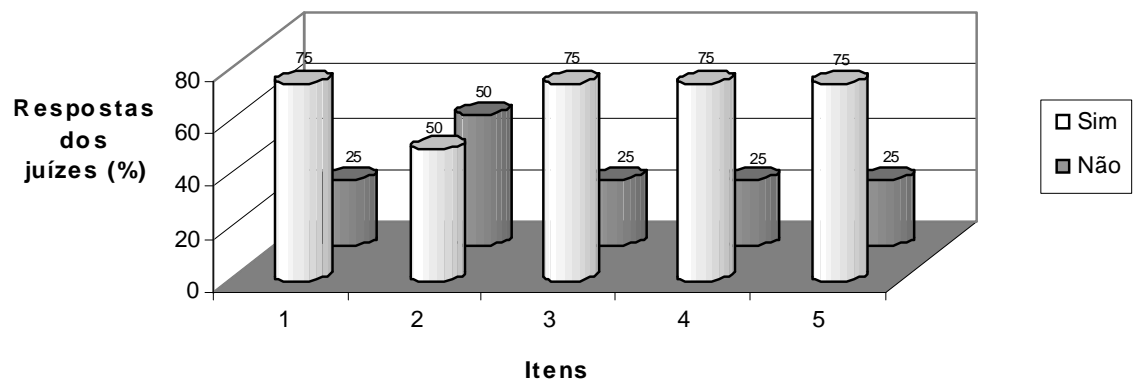

Gráfico 2. Distribuição das respostas dos juízes em relação à organização, objetividade, clareza, facilidade de leitura e compreensão do conteúdo do Guia de Apoio à Decisão da Entrevista de Enfermagem. Campinas, 2004.

Embora os resultados mostrem que, apenas quanto à objetividade ocorreu diferença estatisticamente significativa $(p<0,001)$ no número de respostas entre os juizes e que um deles apontou mais respostas negativas do que os demais, o total de respostas positivas continuou maior, indicando que os juízes compreenderam a maioria dos itens do instrumento e que o conteúdo desse poderia ser entendido pelos enfermeiros. Sugere-se que o instrumento apresenta validade de conteúdo.

A aplicação do referencial teórico por meio da metodologia da assistência de Enfermagem, constituída pela Entrevista de Enfermagem, exige destacar que, neste estudo limitou-se ao caráter de pesquisa, uma vez que foi realizada a validação do instrumento sem que fosse aplicado à assistência. Portanto, o uso deste instrumento na prática diária do serviço deve suscitar novas necessidades de adaptações, que demandarão, novos estudos de validade inclusive em relação ao referencial teórico.

Para exercer de maneira segura e direcionada qualquer atividade profissional, são necessários instrumentos. Especificamente na enfermagem, é preciso direcionamento, sistematização, organização e embasamento científico ${ }^{(2)}$. Esses requisitos da prática podem encontrar subsídios nas teorias de enfermagem.

Acredita-se que a escolha da teoria foi adequada e os resultados da validação sugerem que as modificações necessárias ao modelo adotado foram realizadas.

\section{CONCLUSÕES}

O referencial teórico de Orem possibilitou organizar no instrumento Entrevista de Enfermagem, as necessidades do paciente de acordo com os Fatores Condicionantes Básicos e Requisitos de Autocuidado. Este instrumento, na forma como foi elaborado, possibilita identificar os déficits de autocuidado e a demanda terapêutica, essenciais para o enfermeiro determinar o sistema de autocuidado que será adotado para manter a vida, a saúde e o bem-estar do paciente.

0 instrumento e seu respectivo guia foram apresentados de forma organizada, objetiva, clara, fácil de ler e ser compreendido, sugerindo que podem ser utilizados na prática da enfermagem de uma unidade de internação por possuírem validade de conteúdo. Além disso, alguns itens e subitens do instrumento apontaram a necessidade de serem reavaliados, devido à avaliação negativa que obtiveram.

\section{CONSIDERAÇÕES FINAIS}

Dentre as dificuldades e limitações deste estudo, pode-se destacar: o fato dos juízes terem competências/experiências distintas; a falta de especificação aos juízes sobre a definição dos termos utilizados como critérios de avaliação (organização, objetividade, clareza, facilidade de leitura e compreensão do conteúdo) e, a carência de estudos de validação sobre o tema para fundamentar as análises e discussões.

0 instrumento pesquisado oferece subsídios para direcionar os enfermeiros a documentar a entrevista de enfermagem, permitindo discussões e pesquisas futuras. Tal instrumento pode ser adaptado às características de cada serviço, e quanto mais evidências surgirem de que descreve adequadamente as particularidades de cada paciente, mais validade the será conferida. Assim, consciente de que o instrumento apresentado não é definitivo, as pesquisadoras sugerem outras avaliações no futuro.

\section{REFERÊNCIAS}

1. Orem DE. Nursing: concepts of practice. $4^{\text {th }}$ ed. Saint Louis (MO): Mosby; 1991.

2. Carraro TE. Marco conceitual: subsídio para a assistência de enfermagem. Rev Cogitare Enferm 1998; 3(2): 105-8.

3. Gutierrez BAO, Soares AVN, Anabuki MH, Nomura FH. Histórico de enfermagem. In: Cianciarullo TI, Gualda DMR, Melleiro MM, Anabuki $\mathrm{MH}$, organizadoras. Sistema de assistência de enfermagem: evolução e tendências. São Paulo (SP): Ícone; 2001. p.131-63.

4. Horta WA. Processo de enfermagem. São Paulo (SP): EPU; 1979.

5. Virgínio NA, Nóbrega MML. Validação de instrumento de coleta de dados de enfermagem para clientes adultos hospitalizados. Rev Bras Enferm 2004 jan-fev; 57(1): 53-6.

6. Waldow VR. Processo de enfermagem: teoria e prática. Rev Gaúcha Enferm 1988 jan; 9(1): 14-22.
7. Anselmi ML, Carvalho EC, Angerami ELS. Histórico de enfermagem: compreensão e utilização teórico-prática. Revista da Escola de Enfermagem da USP, São Paulo 1988 ago; 22(2):181-8.

8. Conselho Federal de Enfermagem. Dispõe sobre a sistematização da assistência de enfermagem - SAE - nas instituições de saúde brasileiras: Resolução nº 272/02. Brasília (DF); 2002. [citado em: 24 out 2004].Disponivel em: URL: http://www.corensp.org.br/resolucoes/ resolucoes.html

9. Gaidzinski RR, Kimura M. Entrevista e exame físico - instrumentos para levantamento de dados. In: Campedelli MC, organizadora. Processo de enfermagem na prática. São Paulo (SP): Ática; 1992. p.66-88.

10. Carraro TE. Da metodologia da assistência de enfermagem: sua elaboração e implementação na prática. In: Westphalen MEA, Carraro 
Hermida PMV, Araújo IEM.

$\mathrm{TE}$, organizadoras. Metodologias para a assistência de enfermagem: teorizações, modelos e subsídios para a prática. Goiânia (GO): Editora AB; 2001. p.17-27.

11. Maria VLR, Dias AMC, Shiotsu CH, Farias FAC. Sistematização da assistência de enfermagem no Instituto "Dante Pazzanese" de cardiologia: relato de experiência. Rev Esc Enferm USP 1987 jun; 21(n.esp): 77-87.

12. Foster PC, Janssens NP. Dorothea E. Orem. In: George JB. Nursing Theories: the base for professional nursing practice. New Jersey
(USA): Prentice-Hall; 1980. p.90-106.

13. Contandriopoulos AP, Champagne F, Potvin L, Denis JL, Boyle P. Saber preparar uma pesquisa. 2a ed. São Paulo (SP): Hucitec; 1997.

14. Polit DF, Hungler BP. Nursing research: principles and methods. $5^{\text {th }}$ ed. Philadelphia (USA): Lippincott Company; 1995.

15. Lobiondo-Wood G, Haber J. Confiabilidade e validade. In: LobiondoWood G, Haber J. Pesquisa em enfermagem: métodos, avaliação crítica e utilização. $4^{a}$ ed. Rio de Janeiro: Guanabara-Koogan; 2001. p.186-99. 